\title{
ORIGINAL RESEARCH \\ Differentiation Between Paraclinoid and Cavernous Sinus Aneurysms with Contrast- Enhanced 3D Constructive Interference in Steady- State MR Imaging
}

T. Hirai

Y. Kai

M. Morioka

S. Yano

M. Kitajima

H. Fukuoka

A. Sasao

R. Murakami

Y. Nakayama

K. Awai

R. Toya

M. Akter

Y. Korogi

J. Kuratsu

Y. Yamashita

BACKGROUND AND PURPOSE: Differentiation between paraclinoid and cavernous sinus aneurysms of the internal carotid artery (ICA) is critical when considering treatment options. The purpose of this study was to determine whether contrast-enhanced (CE) 3D constructive interference in steady state (CISS) MR imaging is useful to differentiate between paraclinoid and cavernous sinus aneurysms.

MATERIALS AND METHODS: This study included 11 aneurysms in 10 consecutive female patients, ranging from 52 to 66 years of age. All aneurysms were adjacent to the anterior clinoid process. After conventional and CE 3D-CISS imaging on a 1.5T MR imaging unit, all patients underwent surgery, and the relationship between the aneurysms and the dura was confirmed. Two neuroradiologists evaluated the location of the aneurysms on CE 3D-CISS images and classified them as intradural, partially intradural, and extradural aneurysms. Operative findings were used as a reference standard. To understand the imaging characteristics, we assessed the boundary and signal intensity of the cavernous sinus, CSF, and carotid artery on the side contralateral to the lesion.

RESULTS: Operative findings disclosed that 5 aneurysms were intradural and 6 were extradural. All except 2 were accurately assessed with CE 3D-CISS imaging. One intradural aneurysm adjacent to a large cavernous aneurysm and 1 cavernous giant aneurysm were assessed as partially intradural. On CE 3D-CISS images, the boundary between the CSF, cavernous sinus, and carotid artery was identified by high signal-intensity contrast in all cases.

CONCLUSION: CE 3D-CISS MR imaging is useful for the differentiation between paraclinoid and cavernous sinus aneurysms.

D ifferentiation between paraclinoid and cavernous sinus aneurysms of the internal carotid artery (ICA) is critical when considering treatment options. Paraclinoid carotid aneurysms pose the risk for subarachnoid hemorrhage (SAH) and may be considered for treatment. On the other hand, cavernous sinus aneurysms pose little or no risk for hemorrhage and are usually followed in asymptomatic patients. For the appropriate management of patients with SAH and multiple intracranial aneurysms, it is important to determine whether the aneurysms are located in the intradural portion.

Traditional anatomic landmarks on digital subtraction angiograms for discrimination between paraclinoid and cavernous sinus aneurysms are the origin of the ophthalmic artery ${ }^{1}$ and the anterior clinoid process. ${ }^{2}$ However, these landmarks are not always reliable for making the distinction. ${ }^{3,4}$ Although some researchers reported the usefulness of CT angiography for this issue, ${ }^{3,4}$ the landmarks are indirect signs, and the accurate site at which the ICA penetrates the dura mater is not clearly understood.

3D constructive interference in steady state (3D-CISS) MR imaging can provide high-resolution images with good con-

Received February 12, 2007; accepted after revision May 24.

From the Departments of Diagnostic Radiology (T.H., M.K., H.F., A.S., Y.N., K.A., M.A., Y.Y.), Neurosurgery (Y.K., M.M., S.Y., J.K.), and Radiation Oncology (R.M., R.T.), Graduate School of Medical Sciences, Kumamoto University, Kumamoto, Japan; and Department of Radiology (Y.K.), University of Occupational and Environmental Health, School of Medicine, Kitakyushu, Japan.

Please address correspondence to Toshinori Hirai, MD, Department of Diagnostic Radiology, Graduate School of Medical Sciences, Kumamoto University, 1-1-1 Honjo, Kumamoto 860-8556 Japan; e-mail: t-hirai@kumamoto-u.ac.jp

DOl 10.3174/ajnr.A0756

trast between the CSF and solid structures; they facilitate evaluation of acoustic neurinomas, epidermoids, ventricular lesions, and syringomyelia. ${ }^{5-10}$ Although $3 \mathrm{D}$-CISS imaging is useful for demonstrating cranial nerves in the cistern, contrast-enhanced (CE) 3D-CISS imaging yields good visualization of cranial nerves in the cavernous sinuses. ${ }^{11} \mathrm{CE}$ 3D-CISS imaging may demonstrate the site at which the ICA penetrates the dura mater; however, to date, the usefulness of $\mathrm{CE} 3 \mathrm{D}$ CISS imaging for distinguishing between paraclinoid and cavernous sinus aneurysms has not been assessed.

The purpose of this study was to determine whether CE 3D-CISS MR imaging is useful for the differentiation between paraclinoid and cavernous sinus aneurysms.

\section{Materials and Methods}

\section{Patients}

We prospectively studied conventional MR imaging, MR angiography (MRA), and CE 3D-CISS imaging in 10 consecutive female patients with aneurysms at the ICA adjacent to the anterior clinoid process. Their ages ranged from 52 to 66 years (mean, 59 years); after the MR imaging studies, all underwent surgical treatment. We confirmed the relationship between all aneurysms and the superior wall of the cavernous sinus by surgery.

\section{MR Imaging}

All MR imaging studies were performed with a circularly polarized head coil and a 1.5T superconducting system (Magnetom Vision; Siemens, Erlangen, Germany). Conventional MR imaging, MRA, and CE 3D-CISS sequences were performed during the same procedure. Conventional MR imaging consisted of a sagittal T1-weighted local- 

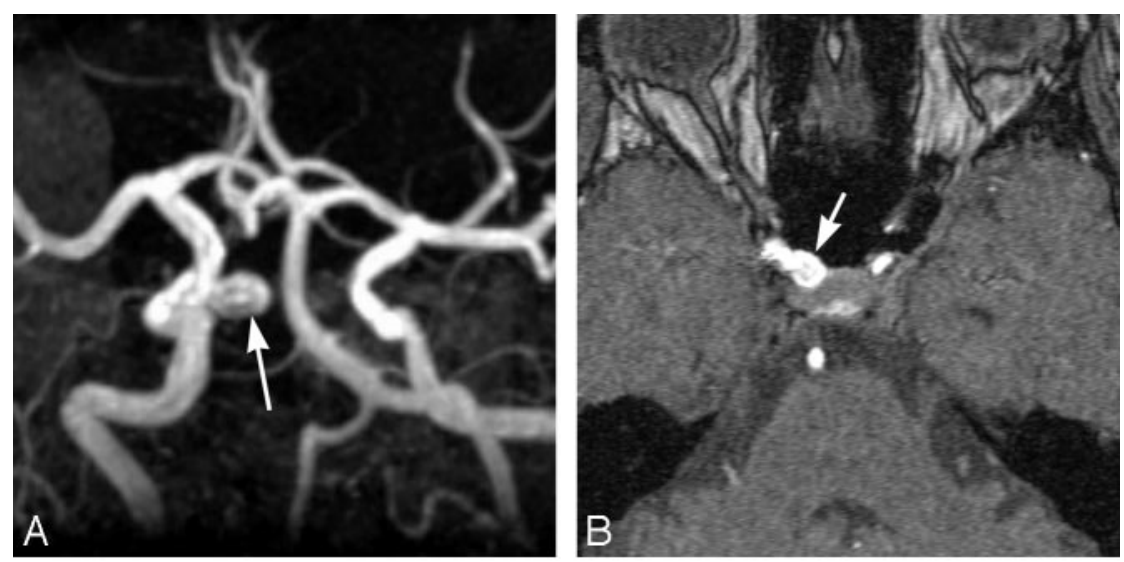

Fig 1. A 50-year-old woman with a right internal carotid aneurysm. $A$ and $B$, Maximum intensity projection $(A)$ and source $(B)$ images of MRA showing a right internal carotid aneurysm (arrow). The distinction between intradural and extradural aneurysms is not clear. $C$ and $D$, Coronal multiplanar reconstruction CE 3D-CISS images revealing that the aneurysm (large arrow) is extradural, located in the cavernous sinus. This observation was confirmed by surgery. The small arrow indicates the boundary between the cavernous sinus and the CSF.
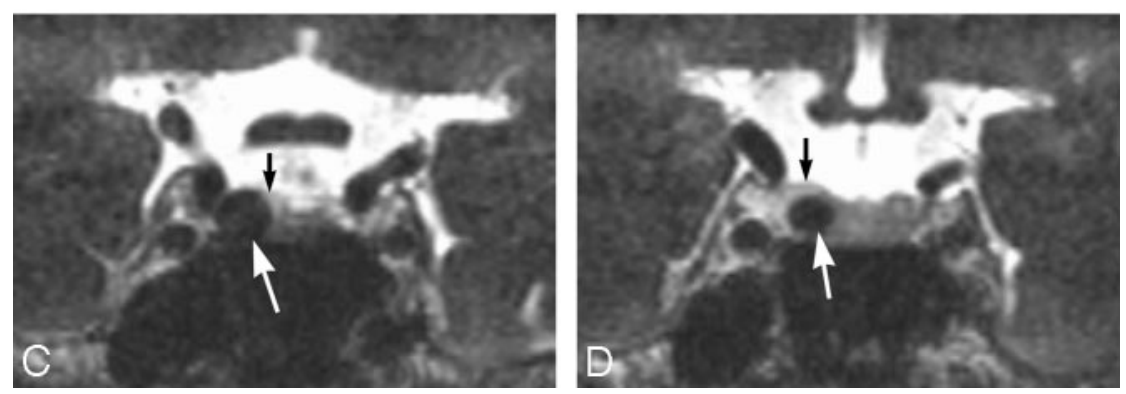

izing sequence (TR/TE/NEX, 15/6/1) and axial T1-weighted (TR/TE/ NEX, 670/14/1), fast spin-echo T2-weighted (TR/TE eff $_{\text {f }} \mathrm{NEX}, 3500 /$ 96/2; echo-train length, 7), and contrast-enhanced T1-weighted sequences. The T1- and T2-weighted sequences were performed at a section thickness of $5 \mathrm{~mm}$ with a 1-mm intersection gap, a $256 \times 256$ matrix, and a 22-cm FOV. Before contrast-enhanced MR imaging studies, 3D time-of-flight MRA was performed with the following parameters: TR/TE/NEX, 32/6/1; $20^{\circ}$ flip angle; 64 -mm slab thickness; 64 partitions; $20-\mathrm{cm}$ FOV; and a $256 \times 512$ matrix. The slabs were placed to include the structures from the intracranial vertebral artery to the A2 branch of the anterior cerebral artery. Cephalad saturation pulses were applied to eliminate venous blood signals. In CE MR imaging studies, gadopentetate dimeglumine $(0.1 \mathrm{mmol} / \mathrm{kg}$ of body weight) was the intravenous contrast agent. Coronal imaging with a CE 3D-CISS sequence (TR/TE/NEX, 12/6.0/1; flip angle, $70^{\circ}$ ) was performed for the evaluation of the bilateral cavernous sinuses; the imaging parameters were the following: matrix size, $224 \times 512$; FOV, $220 \times 220 \times 45 \mathrm{~mm}$; and effective section thickness, $0.7 \mathrm{~mm}$.

\section{Image Analysis}

CE 3D-CISS MR imaging data were analyzed by 2 neuroradiologists by using a multiplanar reconstruction technique. They compared the position of a given point on 1 plane with its position on 2 other perpendicular planes. Each neuroradiologist performed the initial evaluations independently; disagreements regarding final conclusions were resolved by consensus. On the basis of the relationship between the aneurysm and the superior wall of the cavernous sinus on CE 3D-CISS images, the aneurysms were recorded as intradural (the aneurysm was surrounded by CSF), as partially intradural (a portion of the aneurysm was surrounded by CSF), and as extradural (the aneurysm was located in the cavernous sinus). This interpretation was performed in conjunction with reading of the MRA and the multiplanar reconstruction images. The assessments of the neuroradiologists of CE 3D-CISS images were compared with the consensual surgical findings made by 2 neurosurgeons.
To understand the imaging characteristics, we assessed the boundary and signal intensity of the cavernous sinus, CSF, and carotid artery on the side contralateral to the lesion. The boundary between the CSF and the cavernous sinus was evaluated at the superior wall of the cavernous sinus. The 2 neuroradiologists used a 4-point scale, in which grade zero indicated no boundary visible; grade 1 , boundary vaguely visible; grade 2 , boundary visible but with poor delineation; and grade 3 , boundary clearly visible with good delineation.

In our quantitative evaluation of the CE 3D-CISS images, we evaluated the contrast-to-noise ratios (CNRs) of the cavernous sinus and CSF relative to the carotid artery. The CNR of the CSF relative to the cavernous sinus was also assessed. Signal intensity was measured in the CSF, cavernous sinus, and carotid artery; a neuroradiologist placed a circular region of interest on the CE 3D-CISS images. The signal intensity of the background noise was measured outside the head. The size of the region of interest was approximately 20 pixels and that for background noise was approximately 80 pixels. The CNR of the CSF and the cavernous sinus relative to the carotid artery was derived by using the following formulas: $\mathrm{CNR}_{\mathrm{CSF}-\mathrm{CA}}=\left(\mathrm{SI}_{\mathrm{CSF}}-\right.$ $\left.\mathrm{SI}_{\mathrm{CA}}\right) / \mathrm{SD}, \mathrm{CNR}_{\mathrm{CS}-\mathrm{CA}}=\left(\mathrm{SI}_{\mathrm{CS}}-\mathrm{SI}_{\mathrm{CA}}\right) / \mathrm{SD}$, where $\mathrm{SI}_{\mathrm{CSF}}$ is the mean signal intensity of the region of interest within the $\mathrm{CSF} ; \mathrm{SI}_{\mathrm{CS}}$, the mean signal intensity within the cavernous sinus; $\mathrm{SI}_{\mathrm{CA}}$, the mean signal intensity of the carotid artery; and $\mathrm{SD}$, the $\mathrm{SD}$ of noise along the phase-encoding direction. The CNR of the CSF relative to the cavernous sinus was derived by the following formula: $\mathrm{CNR}_{\mathrm{CSF}-\mathrm{CS}}=\left(\mathrm{SI}_{\mathrm{CSF}}\right.$ $\left.-\mathrm{SI}_{\mathrm{CS}}\right) / \mathrm{SD}$.

\section{Results}

We evaluated 11 aneurysms in 10 patients. The maximal diameter of the aneurysms ranged from 4 to $28 \mathrm{~mm}$ (mean, 16 $\mathrm{mm}$ ). Operative findings revealed that 5 aneurysms were intradural and 6 were extradural. All except 2 aneurysms were accurately assessed with CE 3D-CISS imaging (Fig 1). All intradural aneurysms were assessed as intradural $(n=4)$ or 

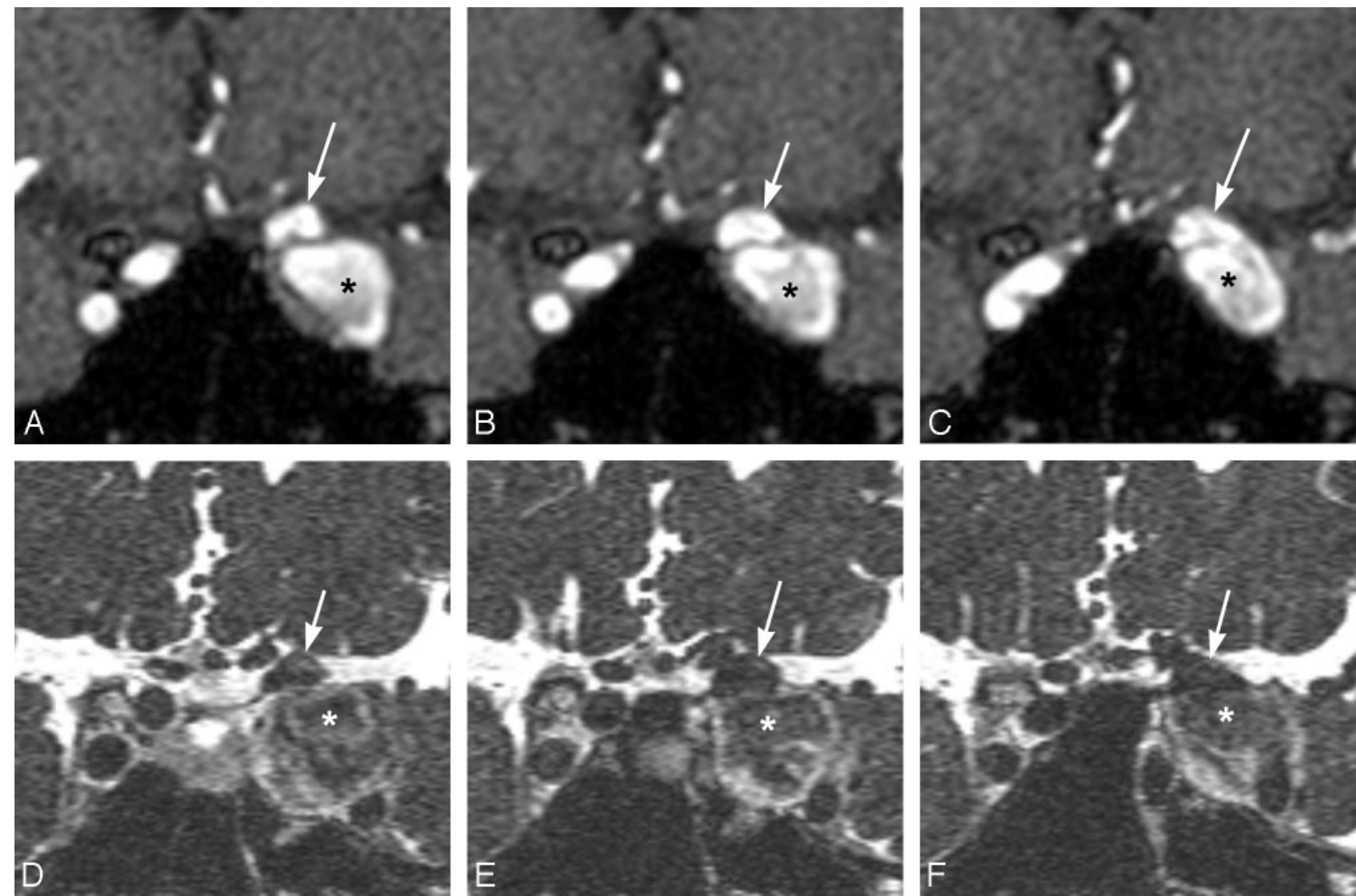

Fig 2. A 59-year-old woman with multiple aneurysms of the left ICA. Coronal multiplanar reconstruction images (A-C) of MRA show 2 left internal carotid aneurysms (arrow, asterisk). On CE 3D-CISS images (D-F), 1 aneurysm (asterisk) is assessed as extradural and the other (arrow), as partially intradural. Operative findings disclosed that they were extradural and intradural aneurysms, respectively

partially intradural $(n=1$, an aneurysm adjacent to a large cavernous aneurysm, Fig 2 ) with CE 3D-CISS imaging. Five of 6 extradural aneurysms were accurately assessed (Fig 1), whereas 1 cavernous giant aneurysm was misinterpreted as partially intradural.

By visual assessment of the boundary between the CSF and the carotid artery and between the cavernous sinus and the carotid artery on CE 3D-CISS images, all the boundaries were scored as grade 3 (Figs 1 and 2). With respect to the boundary between the CSF and cavernous sinus, the mean score was $2.7 \pm 0.48$ (Figs 1 and 2). The mean $\mathrm{CNR}_{\mathrm{CSF}-\mathrm{CA}}, \mathrm{CNR}_{\mathrm{CS}-\mathrm{CA}}$, and $\mathrm{CNR}_{\mathrm{CSF}-\mathrm{CS}}$ were $29.6 \pm 5.2,13.9 \pm 3.1$, and $15.5 \pm 3.1$, respectively.

\section{Discussion}

The dura mater, penetrated by the ICA at the superior portion of the cavernous sinus, consists of 2 fibrous rings. The distal ring is formed by fibers of the superficial dural layer, whereas the proximal ring is related to the deep dural layer. ${ }^{12-14}$ Below the proximal ring, the ICA becomes intracavernous; above the distal ring, it is in the CSF and continuous with its supraclinoid segment. ${ }^{12-14}$ The paraclinoid segment of the carotid artery is defined as the portion between the proximal and distal dural rings, which have a potential space medially, the carotid cave. ${ }^{12,13}$ The carotid cave sometimes communicates with the subarachnoid space. ${ }^{13}$ The superior wall of the cavernous sinus is formed by 2 layers, a smooth superficial dural layer and a thin less-defined deep layer. ${ }^{14}$ In all our patients, we were able to identify the boundary between the CSF and the cavernous sinus on the CE 3D-CISS images. The boundary is thought to be the superior wall of the cavernous sinus.

CE 3D-CISS imaging made it possible to determine accurately the intra- or extradural location of 9 of the 11 aneurysms. On the basis of our qualitative and quantitative studies of the cavernous sinus, CSF, and carotid artery on the side contralateral to the lesion, we attribute its high diagnostic accuracy to its ability to depict small structures with high contrast, at high spatial resolution $(0.7-\mathrm{mm}$-section contiguous images), and on various planes. The cavernous sinus was fully enhanced on CE 3D-CISS images, whereas the carotid artery was not enhanced. Therefore, the contrast between the carotid artery and the cavernous sinus was clearly visualized. In addition, the contrast of the CSF relative to the cavernous sinus was sufficient enough to interpret the boundary between the 2 spaces. According to Yagi et al, ${ }^{11}$ who reported enhancement in the cavernous sinus on CE 3D-CISS images, the cranial nerves in the cavernous sinuses were clearly observed. Shigematsu et $\mathrm{al}^{15}$ reported that although 3D-CISS images yielded heavily T2-weighted sequences, 3D-CISS sequences demonstrated increased contrast as the concentration of gadoliniumbased contrast agents increased. On CE 3D-CISS images, the signal intensity in the cavernous sinuses was not as high as that in the CSF. Although the superior wall was less clear than the lateral wall of the cavernous sinus, the boundary between the $\mathrm{CSF}$ and the cavernous sinus was clearly visualized. The excel- 
lent distinction of these structures on CE 3D-CISS images contributed to our results.

On CE 3D-CISS images, multiple aneurysms adjacent to the anterior clinoid process or a giant aneurysm render accurate diagnosis difficult. In our patient with a giant cavernous sinus aneurysm, high signal intensity from blood in the cavernous sinus was hardly observed, and it was difficult to differentiate the superior wall of the cavernous sinus from the aneurysmal wall. This difficulty may have led to misinterpretation of the location of the aneurysm. Although we did not evaluate the carotid cave itself on CE 3D-CISS imaging in this study, it did not appear that this space could be identified. If a small aneurysm was to efface the CSF or reside in the carotid cave, one might interpret the aneurysm as extradural by this method. Because such limitations attach to CE 3D-CISS imaging, careful interpretation is necessary.

There are some limitations to our study. First, because 3DCISS sequences were obtained only after contrast enhancement in this study, we may not be able to decide whether contrast enhancement is always necessary. It is generally difficult to identify the carotid artery and cranial nerves in the cavernous sinuses on 3D-CISS imaging without contrast enhancement, ${ }^{11}$ and we believe that contrast enhancement is needed for all patients with suspected paraclinoid or cavernous sinus aneurysms. For the supraclinoid aneurysms, however, 3D-CISS imaging without contrast enhancement may depict the aneurysm surrounded by CSF. Second, our study population was relatively small. However, our results are promising for further studies with large populations.

\section{Conclusion}

CE 3D-CISS imaging yields clear visualization of the carotid artery in the cavernous sinus and the boundary between the CSF and cavernous sinus. This imaging technique is useful for the differentiation between paraclinoid and cavernous sinus aneurysms.

\section{References}

1. Punt J. Some observations on aneurysms of the proximal internal carotid artery. J Neurosurg 1979;51:151-54

2. Taptas JN. Intradural and extradural ICA. J Neurosurg 1979;51:877-78

3. Murayama Y, Sakurama K, Satoh K, et al. Identification of the carotid artery dural ring by using three-dimensional computerized tomography angiography: technical note. J Neurosurg 2001;95:533-36

4. Gonzalez LF, Walker MT, Zabramski JM, et al. Distinction between paraclinoid and cavernous sinus aneurysms with computed tomographic angiography. Neurosurgery 2003;52:1131-37

5. Casselman JW, Kuhweide R, Deimling M, et al. Constructive interference in steady state-3DFT MR imaging of the inner ear and cerebellopontine angle. AJNR Am J Neuroradiol 1993;14:47-57

6. Stuckey SL, Harris AJ, Mannolini SM, et al. Detection of acoustic schwannoma: use of constructive interference in steady state three-dimensional MR. AJNR Am J Neuroradiol 1996;17:1219-25

7. Ikushima I, Korogi Y, Hirai T, et al. MR of epidermoids with a variety of pulse sequences. AJNR Am J Neuroradiol 1997;18:1359-63

8. Yang D, Korogi Y, Ushio Y, et al. Increased conspicuity of intraventricular lesions revealed by three-dimensional constructive interference in steady state sequences. AJNR Am J Neuroradiol 2000;21:1070-72

9. Govindappa SS, Narayanan JP, Krishnamoorthy VM, et al. Improved detection of intraventricular cysticercal cysts with the use of three-dimensional constructive interference in steady state MR sequences. AJNR Am J Neuroradiol 2000;21:679-84

10. Hirai T, Korogi Y, Shigematsu Y, et al. Evaluation of syringomyelia with threedimensional constructive interference in a steady state (CISS) sequence. $J$ Magn Reson Imaging 2000;11:120-26

11. Yagi A, Sato N, Taketomi A, et al. Normal cranial nerves in the cavernous sinuses: contrast-enhanced three-dimensional constructive interference in steady state MR imaging. AJNR Am J Neuroradiol 2005;26:946-50

12. Kobayashi S, Kyoshima K, Gibo H, et al. Carotid cave aneurysms of the internal carotid artery. J Neurosurg 1989;70:216-21

13. Oikawa S, Kyoshima K, Kobayashi S. Surgical anatomy of the juxta-dural ring area. J Neurosurg 1998;89:250-54

14. Umansky F, Valarezo A, Elidan J. The superior wall of the cavernous sinus: a microanatomical study. J Neurosurg 1994;81:914-20

15. Shigematsu Y, Korogi Y, Hirai T, et al. Contrast-enhanced CISS MRI of vestibular schwannomas: phantom and clinical studies. J Comput Assist Tomogr 1999;23:224-31 